\title{
LBS Position Estimation by Adaptive Selection of Positioning Sensors Based on Requested QoS
}

\author{
Renato Filjar, Lidija Bušić, Saša Dešić, and Darko Huljenić \\ Ericsson Nikola Tesla d.d. Krapinska 45, 10000 Zagreb, Croatia \\ \{renato.filjar, lidija.busic, sasa.desic, \\ darko.huljenic\}@ericsson.com
}

\begin{abstract}
With increasing attractiveness of location-based services (LBS), the need for consistent establishment and deployment of the LBS Quality of Service (QoS) hierarchy is strongly demanded. The position estimation is in the heart of every location-based service. Thus, LBS QoS is primarily concerned with position estimation performance, including position estimation errors and response time, achieved by either single position sensor, or a combination of several position estimation sensors and methods. Common LBS QoS establishment approach consists of either "as-is" (i. e. no-guarantee) or "best-effort" (again no-guarantee, but with some concern) approach. The proposed new solution starts with generic description of LBS QoS and methods for its deployment. As the result a method emerges that utilises position estimation by adaptive selection of positioning sensors based on requested QoS.
\end{abstract}

Keywords: Location Based Services (LBS) QoS Positioning Sensor Selection.

\section{Introduction}

Determination of the most appropriate Location-Based Services (LBS) Quality of Service (QoS) level is identified as one of the major challenges in the LBS development [1]. So far, various LBS QoS determination approaches have been utilised, that usually offers very limited or no guarantees to the end users.

Since the quality of position estimation sets of the foundation of the LBS QoS, it has been a matter of considerable research to rationalise the usage of position estimation and network resources in order to provide reasonable QoS to a wide number of users [2, 3].

Here we propose system architecture and an algorithm for the LBS position estimation by adaptive selection of positioning sensors based on requested QoS that enables provision of reasonable LBS QoS for particular service, and decreases the overstretching of user and network resources.

\section{Problem Description}

Location-Based Services, as a group of telecommunication services, combines robust and accurate positioning with geospatial (location-related) content and telecommunication 
networks in order to enable provision of location-related content and services to users $[2,3]$. In an analogy to the other telecommunication services, the provision of LBS is driven by various levels of Quality of Service (QoS), i. e. different levels of position estimation accuracy and response time, according to requirements of particular service [1]. Accurate and robust estimation of the user position is the foundation concept of every LBS, regardless of its accuracy [2, 3]. Therefore, the parameters describing the LBS QoS are identified and established in relation to position estimation performance and its distribution throughout the elements of network architecture in support of the LBS provision [1]. Existing LBS-related industrial standards [4, 5] define the following key parameters of LBS QoS:

- Horizontal accuracy,

- Vertical accuracy,

- Response time (time between the request for position determination and the position estimate delivery).

Apparently, different position estimation methods will provide various levels of position estimation accuracy (Fig. 1). Satellite position estimation methods (based on GPS, Glonass, Galileo, GNSS and other satellite positioning systems) have already been identified as the most accurate and reliable, within their limits of operation, and thus assumed to be foundation position estimation methods for LBS development. Other methods (mostly mobile network-based, such as CellID, TOA or E-OTD) are assumed to be assistant methods, or methods of the second choice, due to their inferior QoS compared with satellite positioning methods.

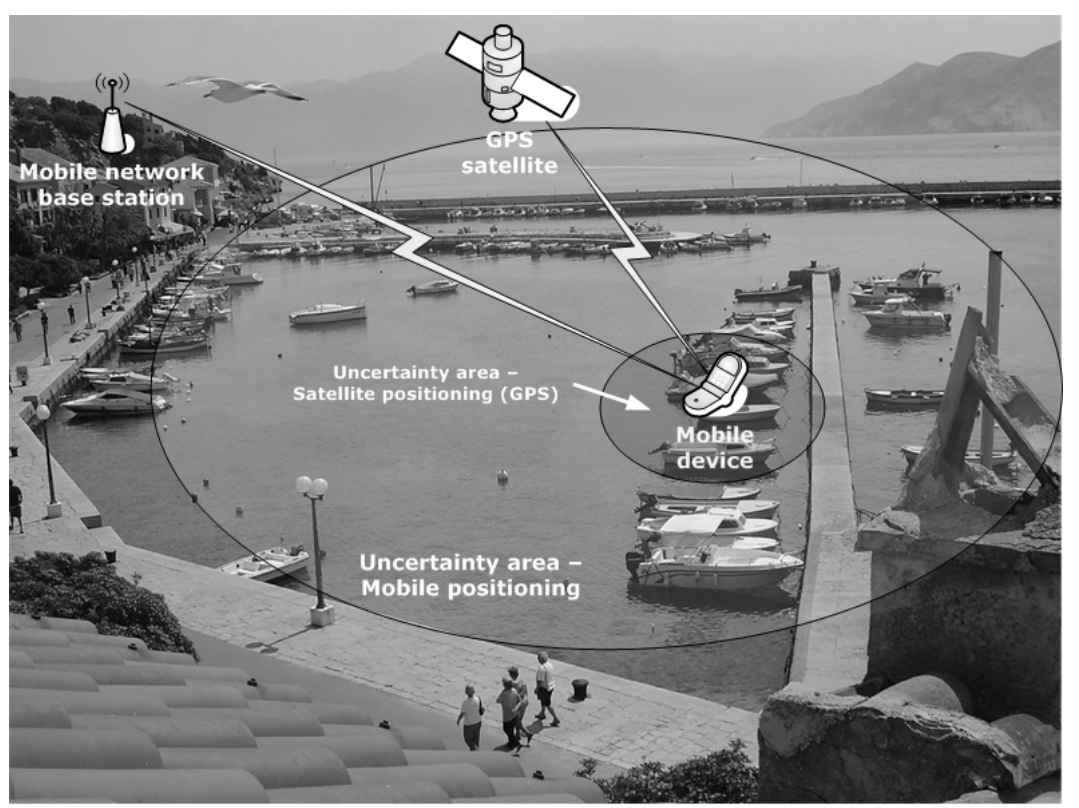

Fig. 1. Different position estimation methods provide different position estimation accuracy (i. e. Quality of Service) 
An adequate level of QoS is set for every LBS service. This means the highest level of position estimation performance is not compulsory for less demanding LBS, such as finding the nearest petrol station. On the other hand, the critical applications, such as notifications of car accidents, require either best-effort or guaranteed highlevel QoS [4].

Position estimation process is based on readings (measurements) conducted on various signals propagating through space. A dedicated device, aimed to perform such measurements and eventually provide the results in a form of either raw signal measurements or as an initial position estimate, is called a position estimation (or positioning) sensor. Positioning sensor is considered an entity deployed within the system architecture for the purpose of either position estimation using particular positioning method, or provision of the additional (assistance and augmentation) data needed for particular positioning method. Various devices may be considered positioning sensors, including, but not limited to: GPS/Galileo/Glonass/GNSS sensor, sensor for WLANbased positioning and network positioning sensors, (comprising various network-based positioning methods, such as Cell ID or E-OTD), augmentation and assistance service for positioning methods (such as A-GPS/GNSS and differential GPS/GNSS).

The position of a (mobile) user can be estimated either from single positioning sensor measurements, or from the combination of more positioning sensor estimates. Although the satellite positioning method (GPS, Glonass, Galileo, GNSS) is widely accepted as the most important and elementary LBS position estimation method $[1,5]$, it cannot be considered a single solution for all LBS positioning needs. However, applicability of satellite positioning systems in critical environments (in-doors, urban and mountainous areas) may be significantly reduced by either degraded position estimation performance (large positioning errors) or complete absence of positioning signal (coverage not available) [6, 7].

In order to tackle disturbing effects on satellite positioning systems, other positioning methods (especially those network-based) should be considered for utilisation in synergy with satellite positioning systems. Such a combination increases the general position estimation performance when both solutions (satellite and network positioning) are available, or provides continuation of position estimation service (although with reduced QoS, especially positioning accuracy) when satellite positioning becomes temporarily un-available. In general, the synergy between satellite positioning and other positioning methods provides more stable and consistent, and less vulnerable LBS QoS compared with the case of satellite positioning system being used alone.

Deployment of combination of several position estimation methods yields the position estimate and positioning error (QoS) estimate, which are obtained in a process called positioning sensor fusion. In positioning sensor fusion, satellite positioning, network-based positioning and other (optional) methods act as the single sources of position estimates (basic positioning sensors and methods). A particular integration method (Kalman filter, neural network, particle filter etc.) should be deployed in order to combine the outcomes of single sources of position estimates, according to the statistical nature of positioning sensor fusion process [6]. Integration methods provide best available position and QoS estimate, which are usually more accurate than those achieved by single position estimation method. In the present concept of positioning sensor fusion, position and QoS estimates are obtained using the come-upon state of methods and resources (position estimation on the as-is basis). Existing concept does 
not utilise the network resources efficiently, since much effort can be put into provision of the best possible QoS (provision of positioning assistance and augmentation, deployment of map-matching algorithm, utilisation of computation-demanding algorithm on network elements) for a service that does not require it. In addition, the endusers are usually being charged for this inefficiency.

\section{Fundamental Concept}

A method for position estimation achieved through adaptive selection of positioning sensors for Location-Based Services (LBS) based on requested QoS is addressed here, with the aim to provide reasonable, appropriate and satisfactorily level of the LBS QoS for particular service in question. The method is based on harmonisation of the particular user and service QoS preferences and capabilities, which provides the LBS QoS level determined by the position estimation performance of the selected positioning sensors. Position and LBS QoS estimates are considered the outcomes of the method for position estimation based on requested QoS. Position estimate is expressed by mandatory (latitude and longitude) and optional (height above the sea level, velocity and azimuth) elements. The LBS QoS estimate is assumed to be expressed by horizontal and vertical position estimation error, and response time. The position estimate is transferred to the LBS application, while the LBS QoS estimate is expected to be utilised internally within the proposed method.

The main concept within the proposed method calls for adaptive selection of positioning sensors, which will be used in position estimation in order to provide the level of LBS QoS both satisfactorily for the end user and not too demanding for the equipment and network resources. The adaptive selection is achieved through the ability of proposed method to suit different conditions of positioning environment (availability of particular positioning sensors and their performance, LBS QoS demands for particular service etc.).

The existence and availability of the following sub-sets of general profiles are necessary prerequisites for proposed method's implementation:

- LBS user profile,

- LBS service profile.

The LBS user profile describes user preferences and provides the list of supported positioning sensors for the particular LBS service invocation. User preferences relates to the choice of preferred positioning method and the willingness to pay for a dedicated positioning process.

LBS service profile determines the minimum requested LBS QoS for actual service in question. It defines the acceptable level of horizontal and vertical positioning accuracy, as well as the response time between the request for positioning and position estimate delivery.

The system architecture in support of the proposed method for position estimation achieved through adaptive selection of positioning sensors for LBS based on requested LBS QoS is presented on Fig 2. The system architecture consists of the client, the LBS application server, the positioning server, and the set of positioning sensors. 


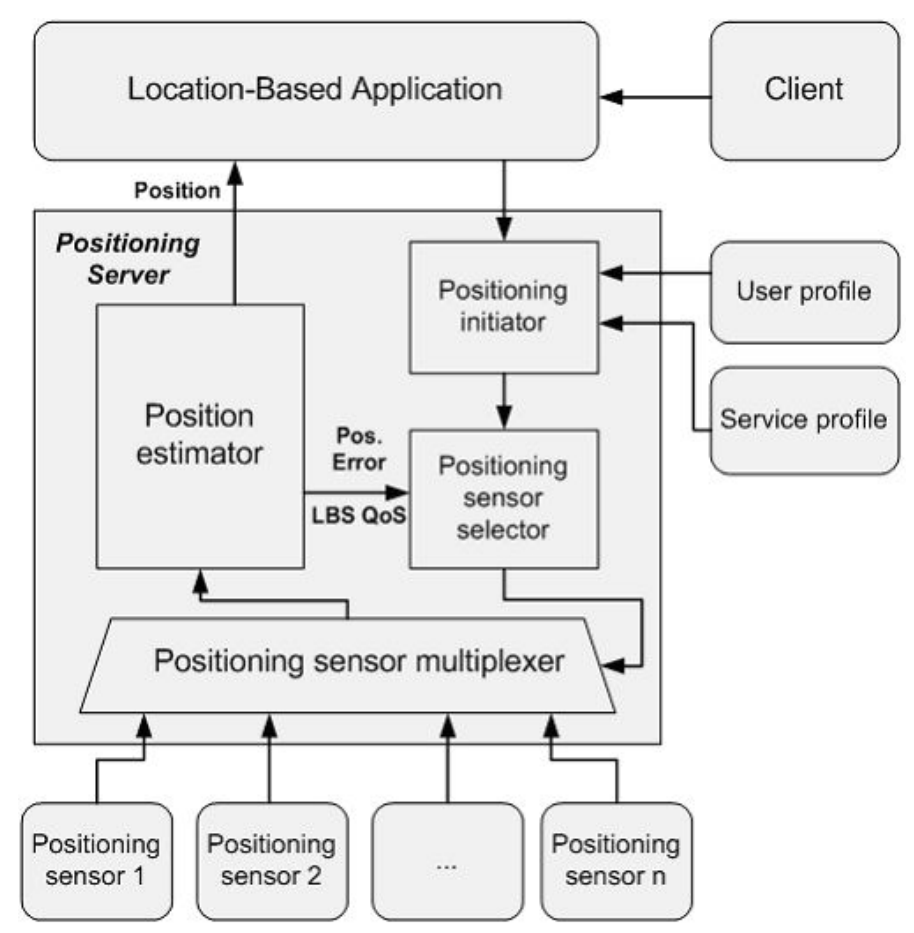

Fig. 2. System architecture in support of the method for adaptive selection of positioning sensors for LBS based on requested QoS

The client serves as the invocator of the LBS service. The Location-Based Application (LBA) handles the LBS invocation requests and position-related content and service provision to the end-users.

Positioning server is in the heart of the system, providing the following dedicated functionalities:

- Positioning sensor multiplexer, for reading the outputs of positioning sensors,

- Positioning initiator, for accepting LBS service initiation requests, and LBS QoS requirements harmonisation based on existing User and Service LBS profiles,

- Positioning sensor selector, for making decisions about activation of particular positioning sensors based on requested LBS QoS,

- Position estimator, aimed to perform positioning sensor fusion, and estimation of position and LBS QoS (position estimation error).

Position and LBS QoS estimation is obtained using different estimation methods that utilise one or the combination of the following:

- Choice of the basic positioning method,

- A method for positioning sensor,

- Choice of Positioning Assistance and Augmentation provision,

- Activation of the context matching algorithm. 
Presented method for adaptive selection of positioning sensors for LBS based on requested QoS is an iterative process in which every iteration results with position and LBS QoS estimates. Control parameter for the iteration process is the congruence between initially requested and estimated LBS QoS.

Application of the method for adaptive selection of positioning sensor for LBS brings considerable benefits, with the list not limited to the following:

- Provision for guaranteed LBS QoS.

- Network resources are used much more efficiently, due to introduction of suitable and reasonable LBS QoS provision, instead of the best effort approach.

- End users are charged for the initiated service and delivered QoS more properly, since the service provides guaranteed QoS.

- A service will be delivered to an end user even though his/her initial set of active sensors does not satisfy QoS requirements.

\section{Adaptive Selection Algorithm}

This chapter presents the positioning sensor adaptive selection algorithm for proposed method. It is based on the current state-of-the-art in mobile communication technologies, especially in relation to up-to-date standardisation in the area of the 3G [5].

The algorithmic representation of the method for adaptive selection of positioning sensors for LBS based on the requested QoS is shown on Fig 3. The aim of the method is to provide position and positioning QoS estimates based on the delivery of suitable and reasonable QoS level for LBS in question, as described in the previous chapter. The algorithm is implemented within the elements of the positioning server.

The iterative process is embedded in positioning procedure of the LBS as follows. After the LBS client initiates particular LBS service, the first iteration of positioning sensor selection is conducted based on requirements for LBS QoS for the service in question, LBS user profile and the set of available positioning sensors. The result is the first set of position and LBS QoS estimates based on obtained list of available positioning sensors. If the obtained position and positioning error estimates failed to reach minimum requirements for service in question, or one or more positioning sensors fail to provide their readings, the iterative procedure follows in which a new set of positioning sensors is to be chosen, and the process repeats until at least the minimum requested LBS QoS is reached, or until all reasonable combinations of positioning sensors are examined without satisfying LBS QoS results. Finally, the whole system architecture is set to its default state, determined by User Profile parameter values, thus maintaining the initial user preferences (initial choice of activated positioning sensors, for instance).

The method starts with the QoS requirements harmonisation and available position estimates collection, both initiated immediately after invocation of the LBS service. The parameters of the LBS user profile and LBS service profile are acquired and combined in order to define the requested LBS QoS for particular service invocation. 


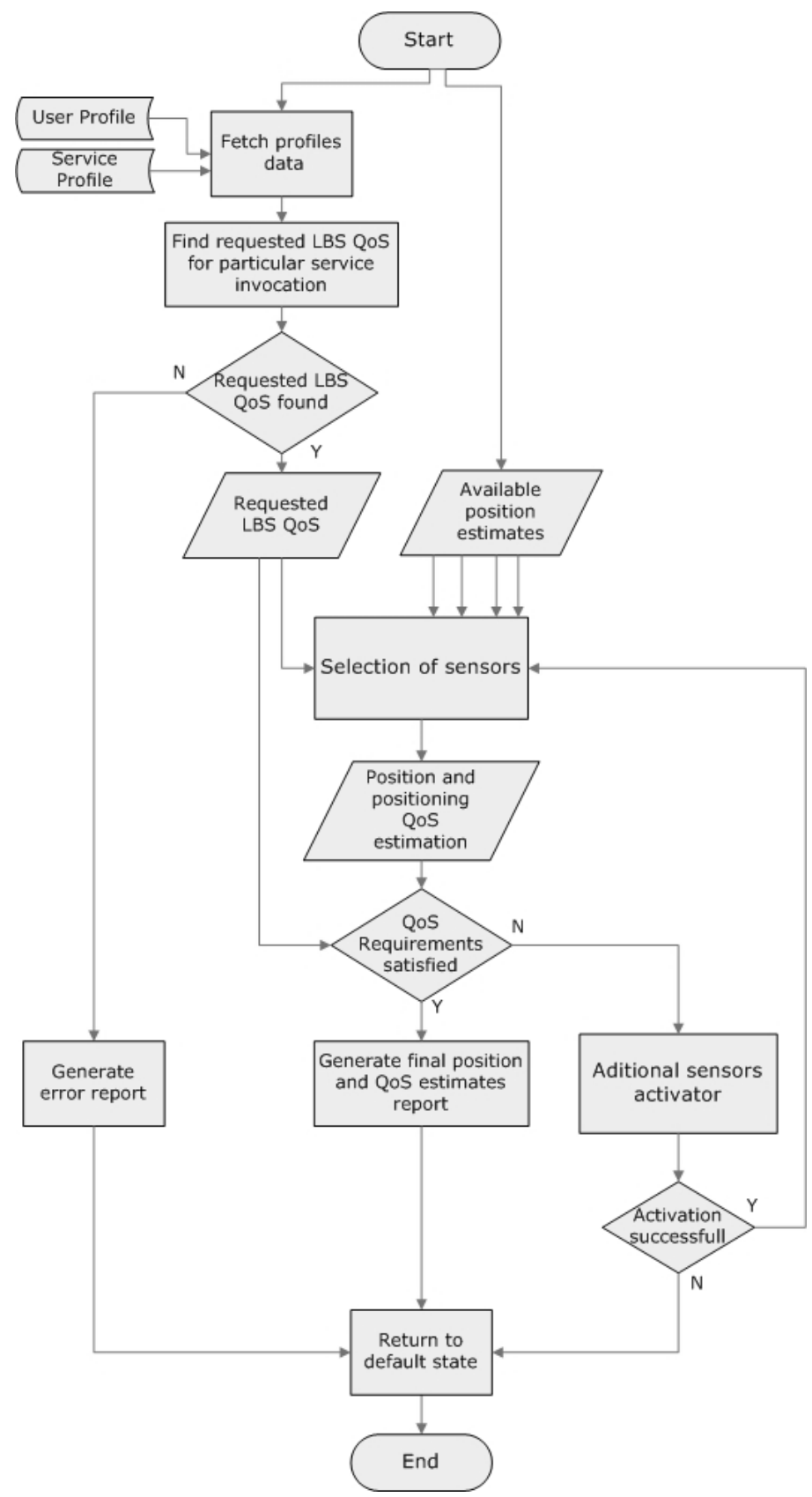

Fig. 3. Sensor selection algorithm supporting the method for adaptive selection of positioning sensors for LBS based on requested QoS 
If the requested LBS QoS is determined for the invoked service, the resulting set of requested LBS QoS parameter values will be forwarded for further processing. If not, an error report will be delivered, which concludes with return to the default state. The default state is determined by user profile.

If the requested LBS QoS is established, it is compared with the available position estimates provided by already active positioning sensors in order to select the firstiteration line of active sensor(s), as seen from the point of view of the requested LBS QoS. Chosen first-iteration line of positioning sensors gives the first-iteration position and positioning error estimation. If those satisfy the requested LBS QoS set earlier, obtained first-iteration position estimate is to be delivered to the LBS application as the final result. Otherwise, the activation of additional positioning sensors (including assistance and augmentation to basic positioning methods, and possible fusion of various positioning sensors) is to be performed.

The main purpose of the Additional Sensors Activator element is to involve more positioning sensors in position estimation procedure, thus allowing for improvement of the actual LBS QoS towards the requested LBS QoS for particular service invocation. The choice of the additional sensors should be based on the list of supported positioning sensors and dedicated LBS QoS error analysis, which ultimately leads to iteration convergence. The LBS QoS error analysis and decision to activate certain positioning sensors should be performed by utilisation of the appropriate optimal control algorithm. Iterations continue until either the requested LBS QoS is reached and position and positioning QoS estimations delivered, or the inability to fulfil requested QoS generates the appropriate service failure report. Finally, the whole system architecture is set to default state, determined by the User Profile.

\section{Conclusion and Future Work}

Proposed method for the LBS position estimation by adaptive selection of positioning sensors based on requested QoS puts an emphasis on introduction and utilisation of the User and the Service Profiles. Instead of exposing the available resources to overdemanding requirements in an attempt to provide the best LBS QoS possible, the proposed method decreases the maximum LBS QoS available to reasonable level that satisfies the end-user, at the same time allowing for more reasonably efficient utilisation of LBS-related resources and ability to serve much more end-users.

Further research will focus on development of advanced positioning sensor selection algorithms based on advanced use of User and Service Profiles, and on achieving the satisfying levels of positioning sensor fusion performance.

\section{Acknowledgements}

The work described in this paper was conducted under the research project New architecture and protocols in converged telecommunication networks (No. 0710362027-2329), approved by the Ministry of Science, Education and Sport, Republic of Croatia. 


\section{References}

1. Filjar, R., Busic, L.: Enhanced LBS Reference Model. In: Proc. of NAV 2007 Conference, p. 8. Westminster, London (2007)

2. Kuepper, A.: Location-based Services: Fundamentals and Operation. John Wiley \& Sons Ltd, Chichester (2005)

3. Steiniger, S., Neun, M., Edwardes, A.: Foundations of Location Based Services (lecture notes). Department of Geography, University of Zurich, Switzerland (accessed on 22 March 2006) (2008), http: / /www.geo.unizh.ch/publications/cartouche/ 1bs_lecturenotes_steinigeretal2006.pdf

4. 3GPP TS 22.071 V7.4.0. Location Services (LCS); Service Description; Stage 1 (2008).

5. 3GPP TS 23.271 V7.7.0. Functional stage 2 description of Location Services (LCS) (Rel 7) (2008)

6. Parkinson, B.W., Spilker Jr., J.J.: Global Positioning System: Theory and Applications, vol. I. AIAA, Washington (1996)

7. Filjar, R., Desic, S., Huljenic, D.: Satellite Positioning for LBS: A Zagreb Field Positioning Performance Study. Journal of Navigation 57, 441-447 (2004)

8. Doucet, A., de Freitas, N., Gordon, N.: Sequential Monte Carlo Methods in Practice. Springer+Business Media, Inc, New York (2001)

9. Schiller, J., Voisard, A.: Location-Based Services. Elsevier, Inc., San Francisco (2004) 